\title{
P04-1-99 Poster session
}

\section{VOLTAGE-GATED SODIUM CHANNEL ACTIVATION TRIGGERS CALCIUM INFLUX AND HIPPOCAMPAL NEURON COMPLEXITY}

\author{
Dina A Gomez, Thomas F Murray \\ Pharmacology, Creighton University, USA
}

Background and significance: After stroke, the molecular mechanisms underlying recovery are similar to the development of the nervous system. During development, neuronal activity regulates both the morphology and connectivity of neurons. Neuronal activity primarily involves voltage-gated sodium channel (VGNaC) activation. Calcium signaling through the $\mathrm{N}$ methyl-D-aspartate receptor (NMDAR) drives morphological changes such as, dendritic arborization, synaptogenesis, and spinogenesis. VGNaC activation influences NMDAR function making this pathway an attractive therapeutic target for stroke recovery.

Hypothesis: Activation of VGNaCs by $\mathrm{PbTx}-2$ augments NMDAR signaling resulting in and increased $\mathrm{Ca} 2+$ influx leading to dendritic arborization and spinogenesis in hippocampal neurons.

Experimental design and Methods: We used the VGNaC gating modifier, brevetoxin ( $\mathrm{PbTx}-2)$, to assess the effects of $\mathrm{VGNaC}$ activation on neuronal morphology. We defined the calcium-signaling pathway triggered by $\mathrm{PbTx}-2$ in a population of hippocampal neurons (HNs), and evaluated $\mathrm{PbTx}$-2-induced effects on dendritic arborization and spinogenesis in organotypic hippocampal slice culture (OHSC).

HNs: cells were prepared from E18 Swiss Webster mouse embryos and plated in 96-well plates. All experiments were run on (10 Days in vitro). A FlexStation2 was used to monitor PbTx-2-induced Ca2+ influx.

OHSC: Slices were prepared from P5, Thy-1 YFP transgenic, mouse pups. Slices were treated with Pbtx-2 18H after plating. A Leica SP8 confocal microscope was used to generate Z-stack images of neurons or dendrites. Imaris-XT software was used to reconstruct 3D-images of the dendritic arbor, run Sholl analysis, and measure spine density.

Results and Conclusion: In HNs, the EC50 for PbTx-2-induced Ca2+ influx was $376 \mathrm{nM}$ (95\% CI, 281-504 nM). The $\mathrm{PbTx}-2$ triggered $\mathrm{Ca} 2+$ influx in $\mathrm{HNs}$ was through the reverse mode of operation of $\mathrm{Na}+/ \mathrm{Ca} 2+$ exchanger, the NMDAR and the L-type Ca2+ channel pathways. In OHSC, four-day treatment with $\mathrm{PbTx}-2(100 \mathrm{nM})$ produced significant increases in dendritic arbor complexity as determined by Sholl analysis $(\mathrm{p}<0.005$, ANOVA). PbTx-2 treatment $(300 \mathrm{nM})$ produced significant increases in spine density $(\mathrm{p}<0.001$, ANOVA). Modifying the gating properties of VGNaC using $\mathrm{PbTx}-2$ enhanced the morphology of hippocampal neurons. Overall, these studies are consistent with the hypothesis that VGNaC activation may represent a novel pharmacological strategy to regulate NMDAR function and aid in recovery poststroke. 